\title{
Avaliação clínica da analgesia pós-operatória proporcionada pelo cetoprofeno associado à morfina pela via intravenosa ou epidural em cadelas submetidas à ovariosalpingohisterectomia
}

\author{
Clinical evaluation of postoperative analgesia provided by \\ ketoprofen associated with intravenous or epidural morphine in \\ bitches undergoing ovariosalpingohysterectomy
}

\author{
Gabriela Carvalho Aquilino Santos ${ }^{1}$; Rosana Maria de Oliveira Clark ${ }^{2 *}$; \\ Roueda Abou Said²; Christiano Midlej Silva Ramos ${ }^{3}$; Alexandre Dias Munhoz ${ }^{2}$
}

\section{Resumo}

\begin{abstract}
A analgesia multimodal refere-se à prática de associar várias classes de analgésicos ou técnicas para atuar em diferentes pontos ao longo da via da dor. Objetivou-se avaliar clinicamente a qualidade da analgesia pós-operatória promovida pelo cetoprofeno, associado ou não à morfina pela via intravenosa ou epidural, em cadelas submetidas à ovariosalpingohisterectomia (OSH). Quarenta cadelas hígidas, pesando $10,7 \pm 6,0 \mathrm{~kg}$, tranquilizadas com acepromazina $\left(0,05 \mathrm{mg}^{-\mathrm{kg}^{-1}}\right.$.iv), induzidas com propofol $(5 \mathrm{mg}$. $\mathrm{kg}^{-1}$.iv) e mantidas sob anestesia inalatória com isoflurano, foram distribuídas em quatro grupos de 10 animais cada. Em Miv e CMiv as cadelas receberam após estabilização da anestesia inalatória, 0,2mg. $\mathrm{kg}^{-1}$ de morfina pela via intravenosa, diluídos em $10 \mathrm{ml}$ de salina; enquanto que os animais de Mep e CMep receberam $0,1 \mathrm{mg} \cdot \mathrm{kg}^{-1}$ de morfina pela via epidural. $\mathrm{O}$ cetoprofeno foi administrado na dose 2,0mg. $\mathrm{kg}^{-1}$.im, nos grupos CMiv e CMep, 30 minutos após a medicação pré-anestésica. Mensuraram-se as frequências cardíaca (FC) e respiratória $(f)$, pressão arterial sistólica (PAS), e temperatura retal (T). O grau de analgesia foi avaliado pelo método cego nas primeiras seis horas após a cirurgia, através de escalas de dor descritivas e escalas compostas por parâmetros fisiológicos e comportamentais. A estatística foi realizada mediante teste de Tukey-Kramer e análise não paramétrica de Kruskal-Wallis, com nível de significância de 5\%. Não houve diferença estatística entre os quatro grupos estudados, em relação à analgesia pós-operatória, $\mathrm{FC}, f$, PAS e T. De acordo com os resultados obtidos, pode-se concluir que o emprego do cetoprofeno, associado à morfina pela via intravenosa ou epidural proporcionou analgesia adequada e segura nas primeiras seis horas de analgesia pós-operatória em cadelas submetidas à OSH, sugerindo não haver potencialização analgésica quando da associação de ambos os agentes.

Palavras-chave: Opióides, AINE, dor, cães
\end{abstract}

\begin{abstract}
Multimodal analgesia refers to the practice of combining multiple analgesic drug classes or techniques to target different points along the pain pathway. The objective of this work was to evaluate clinically if ketoprofen associated or not with intravenous or epidural morphine provided adequate postoperative analgesia in bitches undergoing ovariosalpingohysterectomy (OSH). Forty healthy female dogs,
\end{abstract}

\footnotetext{
1 Mestre do Programa de Pós Graduação em Ciência Animal, Universidade Estadual de Santa Cruz, UESC, Ilhéus, BA. E-mail: gabrielaaquilino@gmail.com

2 Profs. Drs., Dept ${ }^{\circ}$ de Ciências Agrárias e Ambientais, Universidade Estadual de Santa Cruz, UESC, Ilhéus, BA. E-mail: rosanaclark@gmail.com; roueda@hotmail.com.br; admruralvet@ig.com.br

3 Médico Veterinário Autônomo, Itabuna, BA. E-mail: c_midlej@hotmail.com

* Autor para correspondência
} 
weighing $10.7 \pm 6.0 \mathrm{~kg}$, sedated with acepromazine $\left(0.05 \mathrm{mg} \mathrm{kg}^{-1}\right.$. iv), induced with propofol $\left(5 \mathrm{mg} . \mathrm{kg}^{-1}\right.$. iv) and maintained with isoflurane anesthesia, were distributed into four groups of 10 animals each. After stabilization of inhalation anesthesia, the bitches in Miv and CMiv groups received $0.2 \mathrm{mg} \cdot \mathrm{kg}^{-1}$ of morphine intravenously diluted in $10 \mathrm{ml}$ of saline; whereas Mep and CMep groups received $0.1 \mathrm{mg} \cdot \mathrm{kg}^{-1}$ of epidural morphine. Thirty minutes after premedication, $2.0 \mathrm{mg} . \mathrm{kg}^{-1}$. im of ketoprofen was administered in groups CMiv and CMep. Heart and respiratory rate, systolic blood pressure, and rectal temperature were measured. The degree of analgesia was assessed by a blind study in the following 6 hours after surgery, using a descriptive scale and a scale composed by physiologic and behavioral parameters. An statistical analysis was performed using the Tukey-Kramer test and nonparametric Kruskal-Wallis test, with statistical significance of $5 \%$. There was no important difference between the four groups regarding postoperative analgesia, heart and respiratory rate, systolic blood pressure and rectal temperature. According to the results it can be concluded that the use of ketoprofen associated with intravenous or epidural morphine provided adequate and safe analgesia in the first six hours of postoperative in bitches undergoing ovariohysterectomy, suggesting that there was no analgesic potentiation when both agents were combined.

Key words: Opioids, NSAID, pain, dogs

\section{Introdução}

Atualmente, a dor aguda pós-operatória tem sido preocupação crescente na medicina veterinária (MATHEWS, 2002; VALADÃO; DUQUE; FARIAS, 2002). Sabe-se que o processo álgico, sobretudo no paciente cirúrgico, desencadeia uma série de alterações fisiológicas, principalmente neuroendócrinas com efeitos deletérios ao organismo, que podem complicar a recuperação do paciente (FOX et al., 1998; ALVES, 2001; FANTONI; MASTROCINQUE, 2002). Quando se estabelece a dor, na maioria das vezes é muito difícil controlá-la com apenas um agente, necessitando desta forma da associação de terapias envolvendo diferentes classes de analgésicos (LASCELLES; MCFARLAND; SWANN, 2005; QUANDT; LEE; POWELL, 2005).

A terapia multimodal visa à associação de fármacos analgésicos com mecanismos de ação diferentes ou a aplicação de duas ou mais modalidades de tratamento para proporcionar um bom controle da dor, com mínimos efeitos adversos (LAMONT, 2008).

O uso concomitante de anti-inflamatório não esteroidal (AINE) com opióides desempenha um papel significativo no tratamento da dor pós-operatória, principalmente na presença de um processo inflamatório (MATHEWS, 2002;
FLECKNELL, 2008). Vários estudos demonstram que a analgesia peri-operatória, em cadelas submetidas à ovariohisterectomia com a associação de AINE e opióides, proporciona efeitos benéficos e satisfatórios sem a ocorrência de efeitos indesejáveis (MATHEWS, 1996; PIBAROT et al., 1997; MATHEWS, 2002; SLINGSBY; WATERMANPEARSON, 2000; SHIH et al., 2008; MARTINS et al., 2010).

Os AINEs podem proporcionar analgesia por um período de até 24 horas, sendo administrados preferencialmente no período pré-operatório (PIBAROT et al., 1997; MATHEWS; PETTIFER; FOSTER, 2001; MATHEWS, 2002). O cetoprofeno é um derivado do ácido propiônico, sendo considerado um potente agente anti-inflamatório e analgésico, pois inibe ambas isoformas da cicloxigenase (COX1 e COX2), a bradicinina e até certo ponto a lipoxigenase (MATHEWS, 1996; PIBAROT et al., 1997; LASCELLES; MCFARLAND; SWANN, 2005) e está indicado para pacientes que cursam com dor de grau leve a moderado (FANTONI; MASTROCINQUE, 2002).

Estudos demonstram que a dose recomendada deste fármaco de $2,0 \mathrm{mg} / \mathrm{kg}$ é bem tolerada em cães, proporcionando duração de ação de aproximadamente 12 horas, sendo mínimos os efeitos colaterais provocados pelo seu uso 
(PIBAROT et al., 1997; MATHEWS; PETTIFER; FOSTER, 2001; SELMI et al., 2009). Estudo em cães demonstra que este fármaco, quando empregado na pré-medicação pela via intramuscular, se mostrou efetivo para proporcionar recuperação anestésica isenta de fenômenos excitatórios, com adequada sedação, além de analgesia satisfatória durante a avaliação pós-operatória imediata (MATSUDA; FANTONI; FUTEMA, 1999).

Os opióides estão entre os melhores agentes terapêuticos para o controle da dor aguda, devido a sua elevada eficácia e segurança (TAYLOR, 1999; MASTROCINQUE; FANTONI, 2003; LAMONT, 2008). A morfina é um analgésico eficaz, bastante utilizado na Medicina Veterinária, pois não há um limite superior para a analgesia produzida por este agente (QUANDT; LEE; POWELL, 2005). Além disso, ela diminui a resposta álgica decorrente da manipulação cirúrgica e reduz a concentração de anestésico geral necessária para manter o plano anestésico desejado durante o procedimento cirúrgico (VALADÃO; DUQUE; FARIAS, 2002).

A morfina pode ser administrada tanto pela via sistêmica como pela via epidural em cães (POPILSKIS; KOHN; LAURENT, 1993; LASCELLES; MCFARLAND; SWANN, 2005). Entretanto, a principal preocupação sobre seus efeitos, quando administrada pela via intravenosa, é a bradicardia e hipotensão, além da depressão respiratória, podendo facilmente produzir apnéia (HANSEN, 2000). Além disso, a morfina apresenta meia-vida de 1,1 a 1,6 horas quando administrada via sistêmica (PAPICH, 2000), sendo que a duração do efeito analgésico na dose de 0,1 a $0,2 \mathrm{mg} /$ $\mathrm{kg}$, pode perdurar por 3 a 4 horas necessitando muitas vezes de reaplicações, o que pode aumentar a incidência dos efeitos colaterais (FANTONI; MASTROCINQUE, 2002).

As vantagens da administração epidural de opióides incluem analgesia prolongada com mínimos efeitos sistêmicos (JONES, 2001; VALADÃO; DUQUE; FARIAS, 2002; VALVERDE, 2008;
ALMEIDA; ESCOBAR; MAGUILNIK, 2010). A morfina quando administrada pela via epidural na dose de $0,1 \mathrm{mg} / \mathrm{kg}$ promove analgesia que perdura por até 24 horas, porém com período de latência entre 20 a 60 minutos (VALVERDE, 1989; POPILSKIS; KOHN; LAURENT, 1993; JONES, 2001; VALADÃO; DUQUE; FARIAS, 2002).

Diante do exposto, objetivou-se avaliar clinicamente a eficácia do cetoprofeno administrado pela via intramuscular, associado ou não à morfina pela via intravenosa ou pela via epidural, em cadelas submetidas à ovariosalpingohisterectomia (OSH), investigando-se a ocorrência de possíveis efeitos adversos, assim como a qualidade analgésica promovida por estes fármacos para o tratamento da dor pós-operatória imediata.

\section{Material e Métodos}

\section{Animais}

Foram utilizadas 40 cadelas hígidas (ASA I), de diferentes raças, com peso médio de $10,7 \pm 6,0 \mathrm{Kg}$ e idade entre 1 a 8 anos, que foram submetidas à ovariosalpingohisterectomia (OSH), pelo serviço de cirurgia da instituição de origem. A seleção dos animais foi realizada após avaliação clínica prévia, por meio de exame físico e laboratorial como hemograma, contagem de plaquetas, proteínas totais, uréia e creatinina. Os proprietários das cadelas estavam cientes e de acordo com a realização do protocolo em estudo, os quais autorizaram o protocolo experimental mediante o Termo de Ciência e Autorização da Pesquisa. Anteriormente a data da cirurgia os animais foram submetidos a jejum alimentar de 12 horas e hídrico de 2 horas.

\section{Procedimentos anestésicos}

Os animais receberam como medicação préanestésica (MPA) a acepromazina ${ }^{a} 0,2 \%$ na dose de $0,05 \mathrm{mg} / \mathrm{kg}$ pela via intravenosa (IV). Decorridos 15 minutos da MPA, foi realizada a tricotomia da 
região abdominal, da região lombar e da região da veia cefálica sendo esta canulada com cateter intravenoso $22 \mathrm{G}$ para administração de solução de Ringer com Lactato $(10 \mathrm{ml} / \mathrm{kg} / \mathrm{h})$ por todo o período cirúrgico. O cetoprofeno ou placebo (solução salina) foi administrado, pela via intramuscular profunda, após este período de preparo do animal. A indução anestésica foi realizada com propofol $^{\mathrm{b}}$ na dose de $5 \mathrm{mg} / \mathrm{kg} \mathrm{IV}$, ou aquela dose suficiente para intubação orotraqueal. As cadelas foram posicionados sobre colchonete térmico na mesa cirúrgica, intubadas com sonda de Maggill de diâmetro apropriado a sua traquéia, sendo esta conectada ao aparelho de anestesia inalatória em circuito circular semi-fechado, para a manutenção anestésica com isoflurano ${ }^{c}$ diluído em $\mathrm{O}_{2}$ a $100 \%$. Após estabilização da anestesia inalatória, os animais foram posicionados em decúbito esternal com os membros pélvicos estendidos cranialmente (CRUZ et al., 1997) para a punção do espaço epidural, cuja localização foi confirmada através da aspiração de um gota se solução salina posicionada no canhão da agulha. Em seguida à administração epidural, aguardou-se 5 minutos para o posicionamento da cadela em decúbito dorsal e logo foi administrado o opióide ou o placebo pela via intravenosa, conforme descrito nos grupos estudados.

\section{Grupos estudados}

As cadelas foram distribuídas aleatoriamente, por meio de sorteio prévio, e distribuídas em quatro grupos de 10 animais cada. No grupo Miv receberam $1 \mathrm{ml}$ de solução salina IM 30 minutos após a MPA; $0,625 \mathrm{mg} / \mathrm{kg}$ de lidocaína ${ }^{\mathrm{e}}$ a $2 \%$, sem vasoconstritor, diluídos em volume equivalente a $0,26 \mathrm{ml} / \mathrm{kg}$ de solução salina, pela via epidural, no espaço lombossacro, seguida por $0,2 \mathrm{mg} / \mathrm{kg}$ IV de morfina ${ }^{\mathrm{f}}$ diluída em solução salina num volume total de 10 $\mathrm{ml}$, injetada durante 2 minutos após estabilização da anestesia inalatória com isoflurano. No grupo CMiv, os animais receberam $2 \mathrm{mg} / \mathrm{kg}$ IM de cetoprofeno ${ }^{d}$ 30 minutos após a MPA, diluídos em solução salina perfazendo o volume final de $1 \mathrm{ml} ; 0,625 \mathrm{mg} / \mathrm{kg}$ de lidocaína $2 \%$ pela via epidural e $0,2 \mathrm{mg} / \mathrm{kg}$ IV de morfina, conforme descrito anteriormente no grupo Miv. Em Mep administrou-se $1 \mathrm{ml}$ de salina IM; $0,1 \mathrm{mg} / \mathrm{kg}$ de morfina associada a $0,625 \mathrm{mg} / \mathrm{kg}$ de lidocaína $^{\mathrm{e}}$ a $2 \%$, sem vasoconstritor, diluídos em volume equivalente a $0,26 \mathrm{ml} / \mathrm{kg}$ de solução salina, pela via epidural no espaço lombossacro e $10 \mathrm{ml}$ de salina administrada via intravenosa. $\mathrm{O}$ grupo CMep recebeu $2 \mathrm{mg} / \mathrm{kg}$ IM de cetoprofeno após a MPA; morfina associada a lidocaína pela via epidural e 10 $\mathrm{ml}$ de placebo IV, conforme descrito anteriormente em Mep. A utilização da lidocaína em associação a morfina, visou à avaliação da perda dos reflexos motores (relaxamento de cauda e esfíncter anal) o que certificou a correta administração do fármaco no espaço epidural. As cirurgias de $\mathrm{OSH}$ foram realizadas mediante a técnica padrão, pelo mesmo cirurgião.

\section{Parâmetros avaliados}

Durante todos os momentos do estudo foram avaliadas a frequência cardíaca (FC), por meio da auscultação com estetoscópio clínico em batimentos por minuto (bpm); frequência respiratória (f), mensurada pela observação dos movimentos respiratórios em um minuto ( $\mathrm{mpm})$ e temperatura corporal (T) em graus Celsius $\left({ }^{\circ} \mathrm{C}\right)$, através de termômetro clínico pela via retal. Durante a manutenção da anestesia, a pressão arterial sistólica (PAS) foi mensurada pelo método não invasivo através do Doppler vascular, posicionando o manguito na região proximal do rádio, respeitandose uma relação de 0,4 entre a largura do manguito e o comprimento da circunferência do membro.

\section{Avaliação da dor e sedação}

A avaliação pós-operatória da analgesia foi realizada por um único observador (simples cego), através do emprego de três escalas da dor. A escala numérica visual (ENV), mediante escore que varia 
de 0 (sem dor) a 10 (dor crucial ou a pior dor possível), adaptada para avaliação de dor abdominal em cães segundo Mathews, Pettifer e Foster (2001); a escala de análise descritiva, que descartaria a possibilidade de algum sinal de sedação que pudesse mascarar a dor, segundo Lascelles, Butterworth e Waterman (1994) e a escala da dor da Universidade de Melbourn (FIRTH; HALDANE, 1999), que incorpora respostas comportamentais e fisiológicas, incluindo descritivos múltiplos em seis categorias de parâmetros ou comportamentos relacionados a dor, sendo que o escore mínimo é 0 (sem dor) e o somatório é 27 (pior dor possível). Para se avaliar a analgesia, imprimiu-se uma pressão firme ao redor da incisão cirúrgica. Se a cadela apresentasse desconforto em qualquer momento durante o pósoperatório, ou se a pontuação total da escala de Melbourn fosse superior a 9 (SELMI et al., 2009), seria realizada a analgesia resgate com $25 \mathrm{mg} / \mathrm{kg}$ dipirona, pela via intramuscular.

\section{Momentos avaliados}

Os parâmetros cardiorrespiratórios e temperatura corpórea foram avaliados antes da MPA (M0), 15 minutos da MPA (M1), decorridos 15, 30 e 45 minutos (M2, M3 e M4) respectivamente, após indução anestésica com propofol, durante a manutenção anestésica com isoflurano. A analgesia pós-operatória foi avaliada a cada hora após a aplicação da morfina ou placebo pela via sistêmica, perfazendo um total de seis horas (M5, M6, M7, M8, M9 e M10). Foram registrados também o período de tempo da cirurgia, da anestesia, e o momento de extubação orotraqueal. A qualidade da recuperação foi avaliada quanto aos sinais de excitação, presença de vocalização e vômito. Durante a avaliação pósoperatória também foi registrado a aceitação ou não de ração pelos animais.

\section{Análise estatística}

O delineamento estatístico utilizado foi inteiramente casualizado, com 10 repetições e os dados analisados por intermédio do programa computacional SAS (Statistical Analysis System), sendo testada previamente a homogeneidade das variâncias e normalidade dos resíduos e posteriormente, analisados como medidas repetidas no tempo (Repeated Measures), pelo PROC MIXED. As interações significativas foram desdobradas de acordo com os fatores envolvidos. Para os efeitos significativos entre os tratamentos foram realizados testes de comparação de médias, através do comando LSMEANS, utilizando-se o teste de Tukey-Kramer e análise não paramétrica de Kruskal-Wallis. Em todos os procedimentos estatísticos adotou-se 0,05 como níveis crítico de probabilidade para todos os testes realizados.

\section{Resultados e Discussão}

O procedimento cirúrgico resulta em trauma tecidual que varia de intensidade de acordo com o tipo de intervenção, mas que invariavelmente, resulta em algum grau de dor e/ou desconforto para o animal no período pós-operatório imediato (QUANDT; LEE; POWELL, 2005). Embora alguns autores (PIBAROT et al., 1997) incentivem e sustentam a necessidade da utilização de grupos controle, que inicialmente não recebem qualquer terapia analgésica, por questões éticas e humanitárias, decidiu-se não usar um grupo controle sem tratamento. Além do que, acredita-se ser desnecessário submeter outro grupo de animais a tal estímulo, pois já está bem documentado que a OSH causa dor moderada (FOX et al., 1998).

Não houve diferença estatística entre os grupos no tocante ao peso, tempo cirúrgico, duração da anestesia e tempo para extubação após o término no procedimento anestésico (tabela 1). 
Tabela 1. Médias e desvios-padrão referentes ao peso corpóreo $(\mathrm{kg})$, duração da cirurgia, duração da manutenção anestésica, e tempo de extubação orotraqueal, em minutos, de cadelas submetidas à OSH.

\begin{tabular}{ccccc}
\hline & \multicolumn{4}{c}{ Grupos } \\
\cline { 2 - 5 } & Miv & CMiv & Mepi & CMepi \\
\hline Peso (kg) & $12,2 \pm 5,9$ & $8,2 \pm 2,8$ & $10,5 \pm 7,7$ & $12,1 \pm 6,6$ \\
Duração da cirurgia (min) & $31,2 \pm 3,0$ & $30,5 \pm 3,7$ & $33,3 \pm 3,6$ & $28,9 \pm 4,1$ \\
Duração da anestesia (min) & $45,0 \pm 1,0$ & $47,5 \pm 4,7$ & $47,4 \pm 5,6$ & $45,3 \pm 1,9$ \\
Extubação orotraqueal (min) & $11,4 \pm 8,9$ & $7,1 \pm 2,6$ & $9,2 \pm 4,8$ & $10,1 \pm 7,7$ \\
\hline
\end{tabular}

Não houve diferença estatística entre os grupos em nenhuma das avaliações pelo teste de Tukey $(\mathrm{P}>0,05)$. Miv - Morfina intravenosa; CMiv - Cetoprofeno e Morfina intravenosa; Mepi - Morfina epidural; CMepi - Cetoprofeno e Morfina epidural.

Fonte: Elaboração dos autores.

As alterações nos parâmetros respiratórios e cardiovasculares, quando avaliadas isoladamente, nem sempre são bons indicadores de resposta à dor, pois estas podem ser influenciadas por ação de outros fármacos anestésicos (MATHEWS, 2000). É sabido que tanto a acepromazina quanto o propofol podem apresentar alguns efeitos indesejáveis como a hipotensão e a depressão cardiorrespiratória, respectivamente (HALL; CLARKE, 1991; FANTONI; MASTROCINQUE, 2002). Em relação ao protocolo anestésico utilizado para a realização da OSH, não houve influência destes fármacos nos parâmetros fisiológicos, pois não foi observada diferença estatística $(\mathrm{P}>0,05)$ entre os grupos estudados, em relação às frequências cardíaca e respiratória e nem sobre a pressão arterial durante a manutenção anestésica, não havendo, portanto, efeito do tratamento, sobre os grupos estudados.

Ao se analisar os dados da temperatura corpórea, não foi observado diferença estatística entre os momentos para cada grupo. Semelhante aos estudos de Cassu et al. (2008), apesar da aparente redução da temperatura ao longo do tempo, nenhum animal apresentou hipotermia acentuada, com a temperatura mínima registrada em $37,4^{\circ} \mathrm{C}$, não havendo, portanto, importância clínica relevante neste achado. No que se refere à qualidade da recuperação anestésica, alguns sinais de excitação podem ocorrer, como por exemplo, a vocalização que pode ser uma manifestação de delírio pós-anestésico, disforia secundária a administração de opióides, ansiedade ou medo (MATHEWS, 2000). Neste estudo, alguns animais apresentaram vocalização, principalmente após extubação orotraqueal. No entanto, não foi um achado significativo entre os grupos. Nenhum animal apresentou vômito durante a recuperação anestésica, contrastando com a literatura, onde afirma que a morfina epidural atua em zonas quimiorreceptoras do cérebro, podendo desencadear náuseas e vômito (HANSEN, 2000).

Ao se analisar um animal que possivelmente está sentindo dor, alguns aspectos como alimentação e função (atividade) do mesmo devem ser considerados. Os processos álgicos resultam em uma série de alterações fisiológicas como diminuição da ingestão de água e comida, levando à perda de peso, catabolismo protéico e até desidratação (FANTONI; MASTROCINQUE, 2002). Foi oferecido ração seca para os animais durante as seis horas de avaliação analgésica na recuperação anestésica. Observou-se que na sexta hora da avaliação analgésica (M10), 60\% das cadelas que receberam cetoprofeno associada à morfina sistêmica (GII) ou epidural (GIV) ingeriram o alimento, enquanto que em GIII houve $40 \%$ e em GI apenas $10 \%$ de aceitação da ração. Resultado similar foi observado por Oliva et al. (2004), em que constataram que $100 \%$ dos animais que receberam cetoprofeno ingeriram alimento durante as primeiras 24 horas após cirurgias ortopédicas.

Diferentes escalas de dor são utilizadas para a avaliação do desconforto pós-operatório 
associado com a ovariosalpingohisterectomia por vários pesquisadores (MATHEWS; PETTIFER; FOSTER, 2001; MASTROCINQUE; FANTONI, 2003; SHIH et al., 2008; SELMI et al., 2009). $\mathrm{Na}$ medicina veterinária, o comportamento é frequentemente avaliado utilizando-se uma escala análoga visual ou uma escala numérica visual (MATHEWS; PETTIFER; FOSTER, 2001), mas alguns pesquisadores demonstraram que estes sistemas de avaliação são fracos indicadores da dor provenientes de traumas cirúrgicos (HARDIE; HANSEN; CARROLL, 1997). Portanto, empregouse além da escala numérica, a escala de análise descritiva da dor, que descartaria a possibilidade de algum sinal de sedação que possa mascarar a dor (LASCELLES; BUTTERWORTH; WATERMAN, 1994), e a escala da dor da Universidade de Melbourn (FIRTH; HALDANE, 1999), a qual é composta por resposta comportamental e fisiológica específica. Com relação aos escores da dor obtidos com o emprego das três escalas (tabela 2), não foram observadas diferenças entre os escores $(P>0,05)$ nos diferentes grupos estudados, não havendo, portanto, efeito de tratamento no comportamento dos animais durante a recuperação anestésica, nem tampouco efeito de tempo e interação nos diferentes momentos para cada tratamento. Apesar de não ser observada diferença estatística entre os grupos no pós-operatório imediato, houve uma tendência de redução do escore ao longo do tempo dos grupos tratados com a morfina epidural. Em relação à escala de análise descritiva da dor, os grupos que receberam morfina pela via epidural, obtiveram pontuação zero em todos os momentos, corroborando com os resultados relatados por Mastrocinque (2005), em cadelas submetidas à $\mathrm{OSH}$, em que o grupo tratado com morfina epidural $(0,1 \mathrm{mg} / \mathrm{kg})$ apresentou menores escores de dor em vários momentos, em comparação aos grupos controle e ao tramadol.
Também foi demonstrado por Neves (2009), que a administração de morfina epidural promoveu analgesia adequada e de longa duração em cadelas submetidas à OSH, apresentando mínimas alterações nas frequências cardíaca e respiratória, sugerindo ser uma opção segura para cães. A presença de um grande número de receptores opióides na substância gelatinosa do corno dorsal da medula espinhal resulta em maior grau de analgesia tanto na intensidade quanto na duração (VALADÃO; DUQUE; FARIAS, 2002).

De acordo com o trabalho de Shih et al. (2008), no qual comparou-se o efeito da buprenorfina e do carprofeno tanto isolados como associados em cadelas submetidas a OSH, foi constatado que a associação do AINE com um opióide, não potencializou a analgesia do mesmo nas primeiras 6 horas de observação. Este resultado corrobora com os achados do presente delineamento, em que o uso do cetoprofeno não influenciou a analgesia promovida pela morfina empregada tanto pela via sistêmica, como pela via epidural, sendo a mesma, semelhante em todos os grupos estudados.

Nas condições da presente pesquisa, concluiuse que os tratamentos empregados foram eficazes em promover uma analgesia de boa qualidade no pós-operatório imediato de cadelas submetidas à OSH. A associação do cetoprofeno com a morfina não potencializou a analgesia proporcionada por este opióide, apesar do emprego da morfina pela via epidural demonstrar uma analgesia aparentemente melhor do que aquela pela via sistêmica. Adicionalmente, não foram detectados efeitos adversos com o emprego desses agentes. 
Tabela 2. Valores médios e desvios-padrão dos escores obtidos com o emprego das escalas Numérica Visual (MATHEWS; PETTIFER; FOSTER, 2001), Escala de Análise Descritiva da Dor (LASCELLES; BUTTERWORTH; WATERMAN, 1994), e da Escala de Contagem Variável de Melbourn (FIRTH; HALDANE, 1999), obtidos durante o período de avaliação pós-operatória nos diferentes grupos, em cadelas submetidas à OSH.

\begin{tabular}{cccccccc}
\hline \multirow{2}{*}{ Escalas } & \multirow{2}{*}{ Grupos } & \multicolumn{7}{c}{ Pós-operatório } \\
\cline { 3 - 7 } & Miv & $1,0 \pm 0,0$ & $1,0 \pm 0,0$ & $1,1 \pm 0,3$ & $1,1 \pm 0,3$ & $1,2 \pm 0,4$ & $1,2 \pm 0,4$ \\
Escala & CMiv & $1,0 \pm 0,0$ & $1,1 \pm 0,3$ & $1,1 \pm 0,3$ & $1,2 \pm 0,4$ & $1,1 \pm 0,5$ & $1,0 \pm 0,6$ \\
Numérica & Mep & $1,0 \pm 0,0$ & $1,0 \pm 0,0$ & $1,0 \pm 0,0$ & $0,9 \pm 0,3$ & $0,9 \pm 0,3$ & $0,7 \pm 0,4$ \\
Visual & CMep & $1,0 \pm 0,0$ & $1,0 \pm 0,0$ & $1,0 \pm 0,0$ & $1,0 \pm 0,0$ & $1,0 \pm 0,0$ & $0,7 \pm 0,4$ \\
\hline Escala de & Miv & $0,0 \pm 0,0$ & $0,0 \pm 0,0$ & $0,1 \pm 0,3$ & $0,1 \pm 0,3$ & $0,2 \pm 0,4$ & $0,2 \pm 0,4$ \\
Análise & CMiv & $0,0 \pm 0,0$ & $0,1 \pm 0,3$ & $0,1 \pm 0,3$ & $0,2 \pm 0,4$ & $0,2 \pm 0,4$ & $0,2 \pm 0,4$ \\
Descritiva & Mep & $0,0 \pm 0,0$ & $0,0 \pm 0,0$ & $0,0 \pm 0,0$ & $0,0 \pm 0,0$ & $0,0 \pm 0,0$ & $0,0 \pm 0,0$ \\
da Dor & CMep & $0,0 \pm 0,0$ & $0,0 \pm 0,0$ & $0,0 \pm 0,0$ & $0,0 \pm 0,0$ & $0,0 \pm 0,0$ & $0,0 \pm 0,0$ \\
\hline Escala de & Miv & $1,5 \pm 1,2$ & $1,2 \pm 0,9$ & $1,0 \pm 1,0$ & $0,9 \pm 1,0$ & $1,1 \pm 0,9$ & $0,8 \pm 1,0$ \\
Contagem & CMiv & $2,1 \pm 1,4$ & $1,7 \pm 1,1$ & $1,2 \pm 1,1$ & $1,2 \pm 1,1$ & $1,4 \pm 1,5$ & $0,8 \pm 1,0$ \\
Variável de & Mep & $0,8 \pm 0,9$ & $0,8 \pm 0,9$ & $0,7 \pm 0,9$ & $0,4 \pm 0,5$ & $0,4 \pm 0,5$ & $0,4 \pm 0,5$ \\
Melbourn & CMep & $0,8 \pm 0,8$ & $0,7 \pm 0,5$ & $0,8 \pm 0,6$ & $0,5 \pm 0,5$ & $0,5 \pm 0,5$ & $0,3 \pm 0,5$ \\
\hline
\end{tabular}

Não houve diferença estatística entre os grupos em nenhuma das três escalas pelo teste de Kruskal-Wallis ( $\mathrm{P}>0,05)$. Miv - Morfina intravenosa; CMiv - Cetoprofeno e Morfina intravenosa; Mepi - Morfina epidural; CMepi - Cetoprofeno e Morfina epidural.

Fonte: Elaboração dos autores.

\section{Fontes de Aquisição}

${ }^{a}$ Acepram 1\% - Laboratórios Univet S.A. - São Paulo, SP.

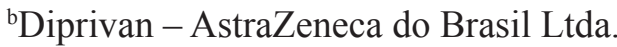

'Isoforine - Cristália Produtos Químicos Farmacêuticos Ltda. - São Paulo, SP.

dDolo Moff-União Química Farmacêutica Nacional S.A. - São Paulo, SP.

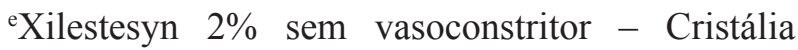
Produtos Químicos Farmacêuticos Ltda. - São Paulo, SP.

${ }^{\mathrm{f} K e t o f e n}$ - Merial Brasil Ltda. - Paulínia, SP.

\section{Comitê de Ética}

Este protocolo experimental foi aprovado pelo Comitê de Ética para o Uso de Animais (CEUA) da Universidade Estadual de Santa Cruz - UESC, processo número 012/2008.

\section{Referências}

ALMEIDA, R. M.; ESCOBAR, A.; MAGUILNIK, S. Comparison of analgesia provided by lidocaine, lidocaine-morphine or lidocaine-tramdol delivered epidurally in dogs following orchiectomy. Veterinary Anaesthesia and Analgesia, Oxford, v. 37, n. 10, p. 542549, 2010.

ALVES, A. S. Emprego do antiinflamatório não esteróide Ketoprofeno na analgesia preemptiva em cães. Ciência Rural, Santa Maria, v. 31, n. 3, p. 439-444, 2001.

CASSU, R. N.; STEVANINI, H.; KANASHIROII, C.; MENEZESII, L. M. B.; LAPOSYI, C. B. Anestesia epidural com lidocaína isolada e associada ao fentanil para realização de ováriossalpingo-histerectomia em cadelas. Arquivo Brasileiro de Medicina Veterinária e Zootecnia, Minas Gerais, v. 60, n. 4, p. 825-831, 2008.

CRUZ, M. L.; LUNA, S. P. L.; CLARK, R. M. O.; MASSONE, F.; CASTRO, G. B. Epidural anaesthesia using lignocaine, bupivacaine or a mixture of lignocaine and bupivacaine in dogs. Veterinary Anaesthesia and Analgesia, Oxford, v. 24, n. 1, p. 30-33, jul. 1997.

FANTONI, D. T.; MASTROCINQUE, S. Fisiopatologia e controle da dor. In: FANTONI, D. T.; CORTOPASSI, S. R. G. Anestesia em cães e gatos. São Paulo: Editora Roca, 2002. cap. 31, p. 323-336. 
FIRTH, A. V.; HALDANE, S. L. Development of scale to evaluate postoperative pain in dogs. Journal of the American Veterinary Medical Association, New York, v. 214, n. 5, p. 651-659, 1999.

FLECKNELL, P. Analgesia from a veterinary perspective. British Journal of Anaesthesia, London, v. 101, n. 1, p. 121-124, 2008.

FOX, S. M.; MELLOR, D. J.; LAWOKO, C. R.; HODGE, H.; FIRTH, E. C. Changes in plasma cortisol concentration in bitches in response to different combinations of halothane and butorphanol, with or without ovariohysterectomy. Research in Veterinary Science, London, v. 65, p. 125-133, 1998.

HALL, L.; CLARKE, K. W. Principles of sedation, analgesia and premetication. In: Veterinary anaesthesia. 9. ed. Londres: Bailiière Tindall, 1991. p. 51-79.

HANSEN, B. D. Acute pain management. Veterinary Clinics of North America: Small Animal Practice, Philadelphia, v. 30, n. 4, p. 899-916, 2000.

HARDIE, E. M.; HANSEN, B. D.; CARROLL, G. S. Behavior after ovariohysterectomy in the dog: what's normal? Applied Animal Behaviour Science, Amsterdam, v. 51, n. 10, p. 111-128, 1997.

JONES, R. S. Epidural analgesia in the dog and cat. The Veterinary Journal, London, v. 161, n. 10, p. 123-131, 2001.

LAMONT, L. A. Multimodal Pain management in veterinary medicine: the physiologic basis of Pharmacologic therapies. Veterinary Clinics of North America Small Animal Practice, Philadelphia, v. 38, n. 10, p. 1173-1186, 2008.

LASCELLES, B. D.; MCFARLAND, J. M.; SWANN, H. guidelines for safe and effective use of NSAIDs in dogs. Veterinary Therapeutics, New Jersey, v. 6, n. 3, p. 237-251, 2005.

LASCELLES, B. D.; BUTTERWORTH, S. J.; WATERMAN, A. E. Postoperative analgesics and sedative effects of carprofen and pethidine in dogs. The Veterinary Record, London, v. 134, n. 8, p. 187-190, 1994.

MARTINS, T. L.; KAHVEGIAN, M. A.; NOELMORGAN, J.; LEON-ROMÁN, M.A.; OTSUKI, D. A.; FANTONI, D. T. Comparison of the effects of tramadol, codeine, and ketoprofen alone or in combination on postoperative pain and on concentrations of blood glucose, serum cortisol, and serum interleukin-6 in dogs undergoing maxillectomy or mandibulectomy. American
Journal of Veterinary Research, New York, v. 71, n. 9, p.1019-1026, sep. 2010.

MASTROCINQUE, S. Avaliação do emprego do tramadol peridural ou sistêmico e da morfina peridural em cadelas submetidas à ovariohisterectomia. 2005. Tese (Doutorado em Cirurgia) - Faculdade de Medicina Veterinária da Universidade de São Paulo, São Paulo.

MASTROCINQUE, S.; FANTONI, D. T. A comparison of preoperative tramadol and morphine for the control of early postoperative pain canine ovariohysterectomy. Veterinary Anaesthesia and Analgesia, Oxford, v. 30, n. 4, p. 220-228, 2003.

MATHEWS, K. A. Non-steroidal anti-inflammatory analgesics: a review of current practice. Journal of Veterinary Emergency and Critical Care, Texas, v. 12, n. 2, p. 89-97, 2002.

Nonsteroidal anti-inflammatory analgesics: indications and contraindications for pain management in dogs and cats. Veterinary Clinics of North America Small Animal Practice, Philadelphia, v. 30, n. 4, p. 783804, 2000.

Nonsteroidal anti-inflammatory analgesics in pain management in dogs and cats. The Canadian Veterinary Journal, Ottawa, v. 37, p. 539-545, 1996.

MATHEWS, K. A.; PETTIFER, G.; FOSTER, R. Safety and efficacy of preoperative administration of meloxicam, compared with that of ketoprofen and butorphanol in dogs undergoing abdominal surgery. American Journal of Veterinary Research, New York, v. 62, n. 6, p. 882888, jun. 2001.

MATSUDA, E. I.; FANTONI, D.T.; FUTEMA, F. Estudo comparativo entre o cetoprofeno e o flunixin meglumine no tratamento da dor pós-opreratória de cães submetidos à cirurgia ortopédica. Clínica Veterinária, São Paulo, ano IV, n. 19, p. 19-22, 1999.

NEVES, C. S. Administração peridural de morfina ou tramadol em cães: efeito analgésico e cardiorespiratório. 2009. Dissertação (Mestrado em Ciência Animal) Universidade do Oeste Paulista, São Paulo.

OLIVA, V. N. L. S.; MAIA, C. A. A.; SILVA, B. M.; SAITO, L. M.; PERRI, S. H. V. Avaliação clínica de diferentes antiinflamatórios não-esteróides na analgesia pós-operatória de cirurgias ortopédicas em cães. A Hora Veterinária, Porto Alegre, ano IX, n. 50, p. 42-54, 2004.

PAPICH, M. G. Pharmacologic considerations for opiate analgesic and nonsteroidal anti-inflammatory drugs. Veterinary Clinics of North America Small Animal Practice, Philadelphia, v. 30, n. 4, p. 815-837, 2000. 
PIBAROT, P.; DUPUIS, J.; GRISNEAUX, E.; CUVELLIEZ, S.; PLANTÉ, J.; BEAUREGARD, G.; BONNEAU, N. H.; BOUFFARD, J.; BLAIS, D. Comparision of ketoprofen, oxymorphone hydrochloride, and butorphanol in the treatment of postoperative pain in dogs. Journal of the American Veterinary Medical Association, New York, v. 211, n. 4, p. 438-444, 1997.

POPILSKIS, S.; KOHN, D. F.; LAURENT, L. Efficacy of epidural morphine versus intravenous morphine for post-thoracotomy pain in dogs. Veterinary Anaesthesia and Analgesia, Oxford, v. 20, p. 21-25, 1993.

QUANDT, J. E.; LEE, J. A.; POWELL, L. L. Analgesia in critically ill patients. The Compendium on Continuing Education Practice Veterinary-Small Animal, v. 27, n. 6, p. 433-445, jun. 2005.

SELMI, A. L.; LINS, B. T.; CESAR, F. B.; FIGUEIREDO, J. P.; DUQUE, J. C. Comparison of the analgesic efficacy of vedaprofeno, carprofen or ketofen after ovariohysterectomy in bitches. Ciência Rural, Santa Maria, v. 39, n. 3, p. 785-790, maio/jun. 2009.

SHIH, A. C.; ROBERTSON, S.; ISAZA, N.; PABLO, L.; DAVIES, W. Comparison between analgesic effects of buprenorphine, carprofen, and buprenorphine with carprofen for canine ovariohysterectomy. Veterinary Anaesthesia and Analgesia, Oxford, v. 35, p. 69-79, 2008.
SLINGSBY, L. S.; WATERMAN-PEARSON, A. E. The post-operative analgesic effects of ketamine after canine ovariohysterectomy - a comparison between pre- or post-operative administration. Research in Veterinary Science, London, v. 69, n. 10, p. 147-152, 2000.

TAYLOR, P. M. Newer analgesics. Veterinary Clinics of North America Small Animal Practice, Philadelphia, v. 29, n. 3, p. 719-735, 1999.

VALADÃO, C. A. A.; DUQUE, J. C.; FARIAS, A. Administração epidural de opióides em cães. Ciência Rural, Santa Maria, v. 32, n. 2, p. 347-355, 2002.

VALVERDE, A. Epidural analgesia and anesthesia in dogs and cats. Veterinary Clinics of North America Small Animal Practice, Philadelphia, v. 38, p. 1205-1230, 2008.

Epidural morphine reduces halothane MAC in dogs. The Canadian Veterinary Journal, Ottawa, v. 36, n. 6, p. 629-632, 1989. 